THE INTERNATIONAL

REVIEW OF RESEARCH IN

OPEN AND DISTANCE LEARNING

\title{
Editorial - Volume 13, Issue Number 5
}
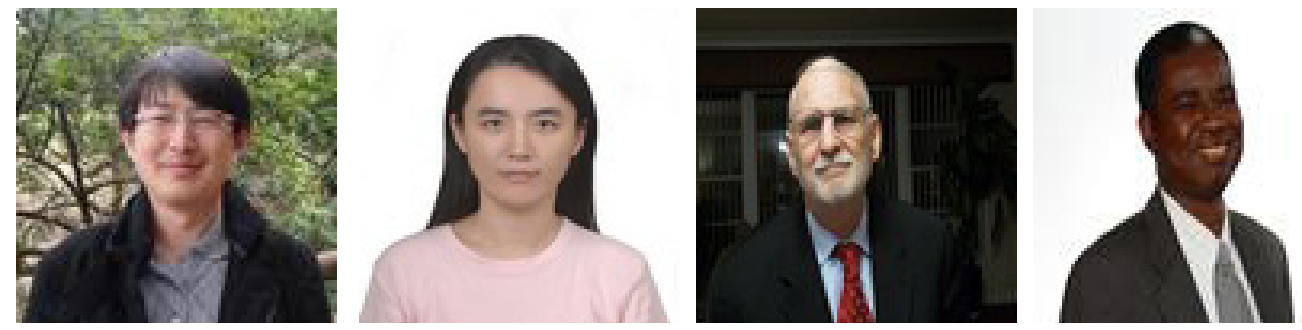

Maiga Chang', Rita Kuo², Gene Loeb ${ }^{3}$ and Bolanle Olaniran ${ }^{4}$ ${ }^{1}$ Athabasca University, Canada

${ }^{2}$ Knowledge Square Inc., Taiwan

${ }^{3}$ Center for Technology and Mental Health of Elderly, USA

${ }^{4}$ Texas Tech University, USA

Technology for enhancing learning has developed rapidly in decades. Teachers can use computer technologies to enrich their learning materials, such as adding images and videos. Abundant teaching materials can be shared on the Web, and learners can easily access plenty of information through the Internet. When learners have questions, they can find answers or solutions by searching the textbook, learning materials, and the Internet. Learners can also ask tutors and peers questions as well as discuss problems with them.

Through sharing and communicating, learning information becomes large and complex. Teachers may experience difficulty managing the volume of teaching materials and assignments, while learners can also experience problems in retrieving and deciphering useful information efficiently and quickly. To solve this problem, information retrieval technology is important for teachers to manage and analyze learning materials and for learners to learn efficiently and effectively.

The purpose of this special issue is to explore how models, theories, and solutions of information retrieval and content analysis can be used in online learning and the benefits users (i.e., teachers and learners) are able to gain from such systems and agents. This special issue covers two aspects of information retrieval techniques - knowledge acquisition and information retrieval application - which can be applied in online learning.

For knowledge acquisition, Dr. Nguyen and Dr. Yang integrate statistics, data mining, and natural language processing techniques to construct a concept tree for the course Introduction to C Programming from text documents in Vietnamese. The method proposed in their article can help tutors reduce the time spent on building the ontology of fifty courses for a 
recommender system which makes suggestions on course selections for students.

Natural language processing can be used to find models in qualitative data analysis also. As such, Dr. Tierney integrates graph theory to accelerate the speed of qualitative interview analysis and to find the categories of analyzed qualitative data. Also, Dr. Wen and colleagues use natural language processing to build an asynchronous question answering system for assisting teachers to respond to learners' messages automatically. The system is able to identify the colloquial nature of the messages and messages mixed with nonquestions in order to eliminate question-irrelevant contents and to find the core questions in the messages. Such a system facilitates communication processes between teachers and learners as well as helps reduce learner's frustrations, attributable to the lack of real-time access to teachers in online learning environments.

Dr. Yu and colleagues, on the other hand, use image processing techniques to analyze instructional videos in order to automatically build an interactive index for learners who choose and watch lessons in videos. Learners can choose a snapshot region or image segment from the instructional video for further in-depth study. The researchers in their experiment found high accuracy of recall rate and precision rate compared to the index made by teachers themselves.

Regarding information retrieval applications, Dr. Tang and colleagues designed a personalized article recommender which can suggest additional readings for learners based on learner characteristics (i.e., interest, background knowledge, job nature, and learning expectation) and article features (e.g., paper topics). Their results show that such a contextaware article recommendation system could be effectively used for inexperienced learners, who are unfamiliar with a particular learning domain or with course content.

Dr. Wang and colleagues use the knowledge-based engine, InfoMap, to draw geometry figures dynamically to help high school geometry students. The accuracy of the figures drawn by the system is near 90\%. Also, Dr. Baldiris and colleagues design two processes to solve the problem of searching for learning objects in distributed learning object repositories and placing the learning objects into adaptive learning design automatically to assist teachers' design work. The first process, Learning Object Repositories SEarcher (LORSE), a distributed learning object searching process, uses two different types of agents - the directory facilitator agent and the specific search agent - to support learners in the learning object search process; the second process, LOOK, a micro-context based positioning process, compares the micro-context of learning objects previously found in the repository and in the curricular structure to decide the best location for the learning object in the adaptive learning design.

Dr. Butakov and colleagues in an attempt to protect student's intellectual property submit student assignments to a plagiarism detection service offered by a third party. This is similar to the approach used by teachers when they see suspicious wording in a student's essay; they will search for the particular sentence(s) with conventional search engines such as Google and Bing. The proposed architecture only submits partial information to exter- 
nal plagiarism detection services to protect students' copyright and to avoid intellectual property violations. Finally, Dr. Cheng and colleagues developed an e-book hub service on a free and open-source cloud computing platform - OpenStack. The e-book hub service is designed to help teachers produce, transform, and manage their e-books to fit the needs of students who are using different platforms including smart phones, tablets, and desktop computers.

\section{Athabasca University $\mathbf{A}$}

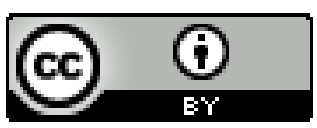

